\title{
Characterization of Focal Liver Lesions using Contrast Enhanced Ultrasound as a First Line Method: a Large Monocentric Experience
}

\author{
Ioan Sporea, Alina Martie, Simona Bota, Roxana Șirli, Alina Popescu, Mirela Dănilă
}

Department of

Gastroenterology and

Hepatology, Victor Babeș

University of Medicine and

Pharmacy

Timișoara, Romania

\author{
Address for correspondence: \\ Roxana Sirli, MD, PhD \\ Department of \\ Gastroenterology and \\ Hepatology, Victor Babeș \\ University of Medicine and \\ Pharmacy \\ Timișoara, Romania
}

roxanasirli@gmail.com

Received: 06.11.2013

Accepted: 19.02.2014

\begin{abstract}
Aim: To present a large monocentric experience in the characterization of focal liver lesions (FLLs) using Contrast Enhanced Ultrasound (CEUS).

Method: A retrospective study was performed in the Gastroenterology and Hepatology Department, Timisoara, including 1100 patients with 1329 FLLs evaluated between September 2009 and January 2013. A CEUS examination was considered conclusive if the FLL respected the typical enhancement pattern as described in the EFSUMB Guidelines.

Results: From the 1329 FLLs, CEUS was conclusive for a specific pathology in 1102 cases (82.9\%). For the differentiation of benign/malignant lesions, CEUS reached a conclusive diagnosis in 1196 (90\%) cases. The percentage of conclusive CEUS examinations was significantly higher in patients without chronic liver disease as compared with those with chronic hepatopathies: $87.3 \%$ vs. $74.4 \%(\mathrm{p}<0.0001)$.

Conclusion: CEUS patterns of enhancement fell into clear cut specific diagnostic patterns in $83 \%$ of the FLLs discovered by US, and into clear cut benign versus malignant patterns in $90 \%$ of the cases. For this reason, we can strongly recommend CEUS as a first line imaging method to characterize FLLs found at US, at least in centers with a good experience in CEUS.
\end{abstract}

Key words: Contrast Enhanced Ultrasound (CEUS) - focal liver lesions (FLLs) - benign/malignant liver lesions.

\section{INTRODUCTION}

Ultrasonography (US) is a simple, inexpensive and useful method for the evaluation of the liver. This method is used by clinicians or by radiologists, but in the last years, other categories of physicians, such as general practitioners, also have begun to use it in daily practice. The quality and performance of US machines differ, but in the end standard US is an accurate tool for the detection of focal liver lesions (FLLs), although with a lower value for their characterization.

During the abdominal examination, FLLs can be detected, incidentally or not, in asymptomatic patients, in patients with known liver disease (especially liver cirrhosis), or in patients with an oncologic history. When a FLL is discovered, it should be further evaluated in order to elucidate its etiology. There are several imaging ways to evaluate these lesions: contrast enhanced ultrasonography (CEUS), contrast enhanced computed-tomography (CE-CT) or contrast enhanced magnetic resonance imaging (CE-MRI), the last two more expensive. In 2004, the European Federation of Societies for Ultrasound in Medicine and Biology (EFSUMB) issued Guidelines and Recommendations concerning the use of CEUS [1] that were revised in 2008 [2] and in 2012 [3]. The last Guidelines were developed in cooperation with the World Federation for Ultrasound in Medicine and Biology (WFUMB), in cooperation with representatives of the Asian Federation of Societies for Ultrasound in Medicine and Biology (AFSUMB), American Institute of Ultrasound in Medicine (AIUM), Australasian Society for Ultrasound in Medicine (ASUM), Latin-American Federation of Societies for Ultrasound in Medicine and Biology (FLAUS) and International Contrast Ultrasound Society (ICUS), so that one can consider that these last Guidelines have universal availability [3].

According to these Guidelines, the vascular enhancement pattern is used to characterize FLL, since almost every type 
of FLL has a particular CEUS appearance. Several studies compared CEUS with CE-CT or CE-MRI, showing similar accuracy for the characterization of some types of FLLs [48]. Considering the data presented in large studies $[4,5]$ and meta-analyses $[7,8]$ that confirm the good results of CEUS for the differential diagnosis of FLLs, this method can be used as a first line imaging method for an accurate diagnosis of a new liver lesion discovered by US examination.

The aim of our study was to retrospectively evaluate, in a center with an extensive experience in CEUS examination of the liver, how relevant is this method in front of a new lesion discovered by US.

\section{PATIENTS AND METHODS}

\section{Patients}

A retrospective study was performed in the Gastroenterology and Hepatology Department in Timisoara, which included 1100 patients evaluated between September 2009 and January 2013. The inclusion criteria were: FLLs detected in patients with or without chronic liver disease which were identified by US, but could not be characterized only by standard US. This category also included FLLs found in patients with a history of neoplasia. The exclusion criteria were: FLLs in patients with or without oncologic history which presented malignant FLLs documented by other methods (CE-CT/CE-MRI, biopsy); FLLs which were not clearly visible on US; FLLs previously assessed by CEUS (hepatocellular carcinoma, HCC or liver metastasis percutaneously treated); patients with NYHA III/ IV heart failure.

All patients agreed to undergo a CEUS examination. The study was approved by the Ethics Committee of the institution in accordance with the Helsinki Declaration of 1975.

\section{Imaging techniques}

All FLLs were evaluated using standard US, Doppler US and CEUS. CEUS was the first contrast imaging method used for the characterization of these lesions. A CEUS examination was considered conclusive if the FLLs respected the typical enhancement pattern stated in the EFSUMB Guidelines [2] and otherwise inconclusive. In inconclusive cases we performed second-line diagnostic procedures (CE-CT, CE-MRI or biopsy) to establish the final diagnosis.

Ultrasonography was performed with a Siemens Acuson S2000 ${ }^{\mathrm{TM}}$ system (Siemens AG, Erlangen, Germany), using a $3.5 \mathrm{MHz}$ convex transducer. The location, the size, the number and the echogenity of the FLLs were reported.

Doppler US technique was performed using the same system in order to observe the tumor vascularization.

Contrast-enhanced US was performed with a convex probe using a low mechanic index (0.09-0.11). The contrast agent used was SonoVue ${ }^{\circledast}$ (Bracco SpA, Milan, Italy), usually $2.4 \mathrm{ml}$, which was injected through a peripheral intravenous cannula of appropriate size, followed by a $10-\mathrm{mL}$ saline flush, as per standard protocol. The lesions' enhancement patterns were studied in 3 phases: arterial (10-30 seconds after injection), portal (30-120 seconds) and late phase ( $>120$ seconds) according to the EFSUMB recommendations $[2,3]$. Each examination lasted about 5 minutes. At the end of examination, the result was reported as conclusive (if the EFSUMB criteria were met) or inconclusive (if the enhancement pattern could not be matched to the EFSUMB guidelines). The first decision as conclusive vs. inconclusive that had to be made was regarding the benign vs. malignant character of the FLL, and secondary regarding the type of lesion (characterization of the lesion). The examinations were performed by four senior gastroenterologists with extensive experience in abdominal US and in liver evaluation by CEUS. Recorded loops of the examinations were revised several times, as needed.

We observed the following types of enhancement pattern as compared to the adjacent liver parenchyma: hyperenhancement, when the FLL was filled more intensely than the surrounding parenchyma; isoenhancement, when the lesion was filled similarly with the surrounding parenchyma; hypoenhancement, when the lesion was filled less than the adjacent parenchyma; rim-like pattern, when the lesion was enhanced only in the periphery, representing more than $25 \%$ of tumor size; wash-in/washout, when the lesion was hyper/isoenhanced in the arterial phase, followed by hypoenhancement in the venous and/or late phases. All examinations were digitally recorded. A CEUS examination was considered conclusive if, following contrast, the FLL had a typical enhancement pattern according to the EFSUMB guidelines [2,3] and otherwise inconclusive. Also, the benign or malignant nature of a FLL was established according to the absence/presence of washout in the portal and/or late phases of examination $[2,3]$. We classified the types of lesions according to the EFSUMB guidelines $[2,3]$.

a) Benign lesions

1. hemangioma: centripetal enhancement pattern in the arterial phase, partial or complete centripetal filling in the portal phase and homogeneous complete enhancement in the late phase (Fig. 1);

2. focal nodular hyperplasia (FNH): early arterial hyperenhancement with typical centrifugal radiating or "spoke-wheel" pattern, followed by complete and homogeneous hyperenhancement pattern in the late arterial and also in the portal phase and iso/hyperenhancement pattern in the late phase;

3. hepatocellular adenoma: early and homogeneous hyperenhancement in the arterial phase, isoenhancement in the portal phase and iso/hypoenhancement in the late phase;

4. focal fatty alterations: the same enhancement pattern as the surrounding liver parenchyma in all phases;

5. liver cysts: no enhancement in any of the vascular phases;

6. regenerative nodule: the same vascular pattern as the surrounding liver in all phases;

7. abscess: rim-like enhancement pattern in the arterial phase, hypo/iso-enhancing rim in the portal phase and hypoenhancing rim in the late phase;

8. hematoma: no enhancement in any of the vascular phases.

\section{b. Malignant lesions}

1. HCC: hyperenhancement in the arterial phase (with/ without unenhanced necrotic areas), isoenhancement (with/without unenhanced areas) in the portal phase, hypo/ isoenhancement pattern in the late phase (Fig. 2); 
2. hypervascular liver metastases: early and complete arterial enhancement, portal hypoenhancement, hypoenhancement or absence of the enhancement in the late phase;

3. hypovascular liver metastases: rim-like enhancement in the arterial phase, hypoenhancement in the portal and late phases;

4. cholangiocarcinoma (CC): peripheral fill-in in the arterial phase, hypoenhancement or absence of enhancement in the portal phase, hypoenhancement or absence of enhancement in the late phase.

\section{Statistical analysis}

Statistical analysis was performed using the MedCalc Software (MedCalc, Belgium). For the numerical variables, mean value and standard deviation were calculated. The Chisquare $\left(\chi^{2}\right)$ test (with Yates' correction for continuity) was used for comparison of two proportions expressed as percentages. 95\% confidence intervals were calculated for each predictive test. A p-value less than 0.05 was regarded as significant for each statistical test.

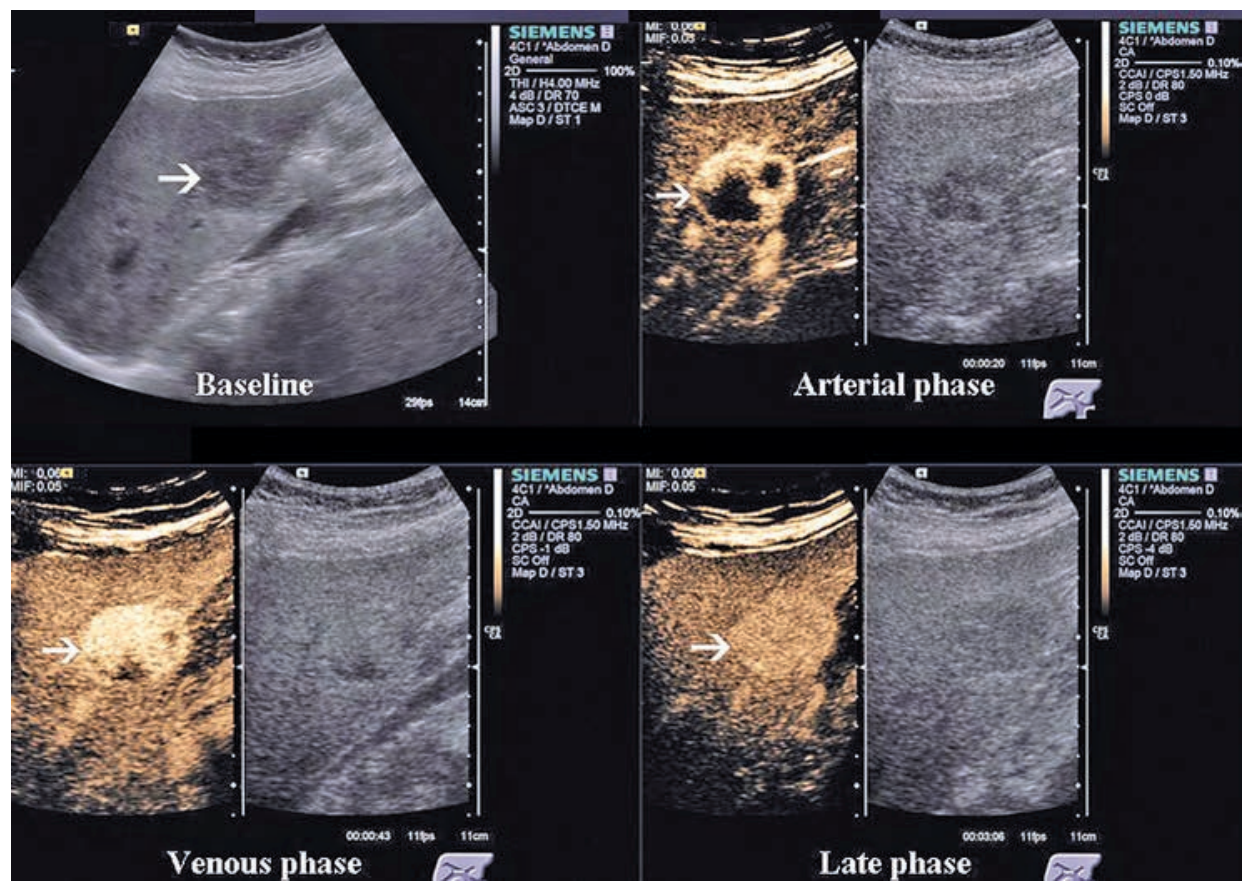

Fig. 1. The enhancement pattern of a hemangioma in CEUS - centripetal rim enhancement pattern in the arterial phase, partial centripetal filling in the venous phase and homogenous complete enhancement in the late phase (no washout - typical for a benign FLL).

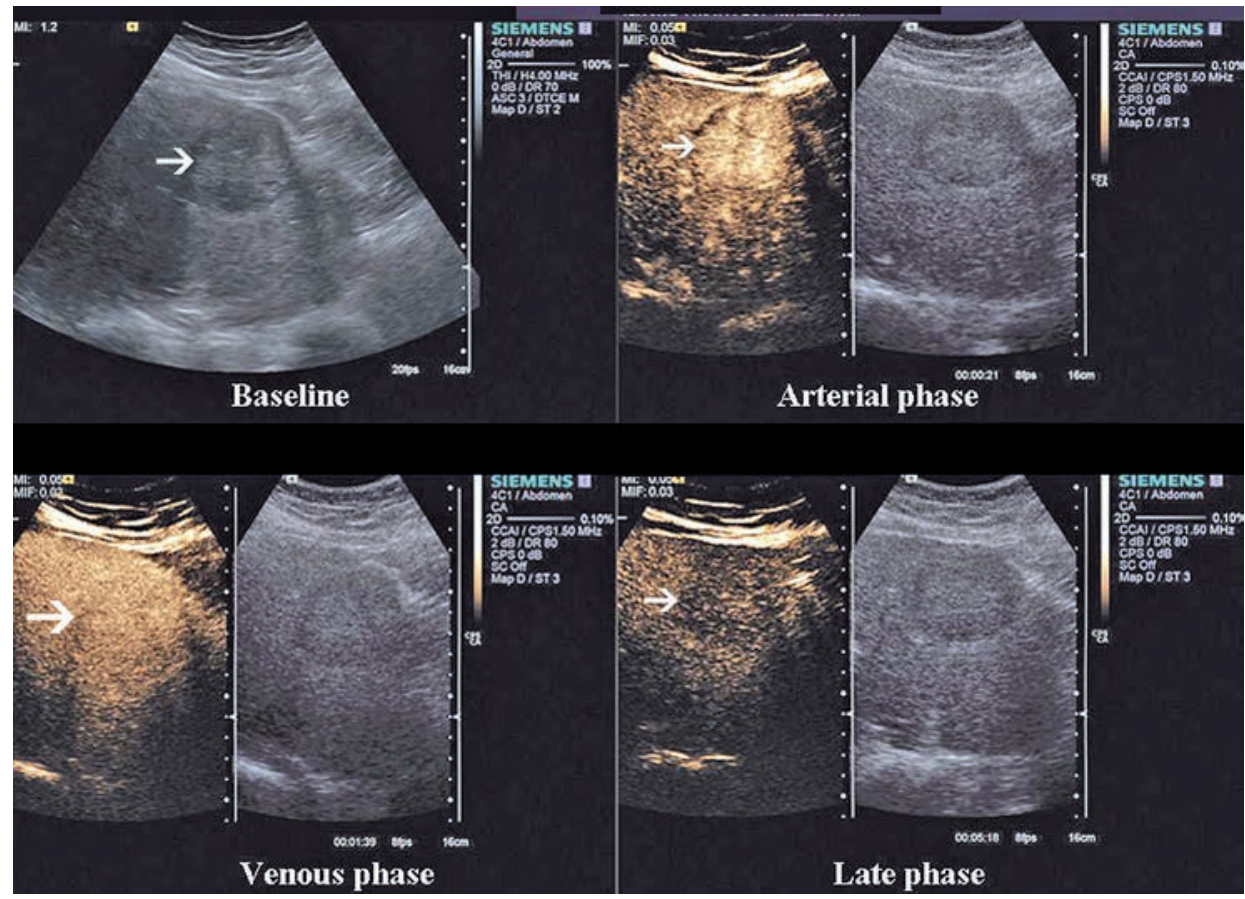

Fig. 2. The enhancement pattern of a HCC: hyperenhancement in the arterial phase, isoenhancement in the portal phase, hypoenhancement in the late phase (washout in the late phase - typical for a malignant lesion). 


\section{RESULTS}

From September 2009 to January 2013 we evaluated 1100 patients with 1329 FLLs (Table I). Out of the 1329 evaluated FLLs, CEUS was conclusive for a apecific type of lesion in 1102 cases $(82.9 \%)$. The proportion of conclusive CEUS examinations was significantly higher in patients without chronic hepatopathies as compared with those with chronic liver disease: $87.3 \%$ vs. $74.4 \%, \mathrm{p}<0.0001$.

For the differentiation between benign and malignant FLLs, CEUS showed a conclusive diagnosis in 1196 (90\%) cases.

According to the size of FLL $(\leq 2 \mathrm{~cm}$ and $>2 \mathrm{~cm})$, CEUS was conclusive for a specific pathology in $291(82.4 \%)$ cases and in $811(83.1 \%)$ cases, respectively. The proportion of conclusive CEUS examinations was similar regarding small and large FLLs: $82.4 \%$ vs. $83.1 \%, \mathrm{p}=0.82$.

Table I. Patients' characteristics

\begin{tabular}{|c|c|}
\hline & Data \\
\hline Median age (range, years) & $58.7(20-92)$ \\
\hline $\begin{array}{l}\text { Gender }(\mathrm{n}, \%) \\
\text { women } \\
\text { men }\end{array}$ & $\begin{array}{l}485(44.1 \%) \\
615(55.9 \%)\end{array}$ \\
\hline Median size of the FLL (range, $\mathrm{cm}$ ) & $4.2(0.5-17)$ \\
\hline $\begin{array}{l}\text { FLL according to the size }(\mathrm{n}, \%) \\
\qquad \begin{array}{l}\leq 2 \mathrm{~cm} \\
>2 \mathrm{~cm}\end{array}\end{array}$ & $\begin{array}{l}353(26.6 \%) \\
976(73.4 \%)\end{array}$ \\
\hline $\begin{array}{l}\text { Number of FLL/patient }(\mathrm{n}, \%) \\
\text { one } \\
\text { two }\end{array}$ & $\begin{array}{l}968(88 \%) \\
66(12 \%)\end{array}$ \\
\hline Benign FLL (n, \%) & $654(49.2 \%)$ \\
\hline Malignant FLL (n, \%) & $675(50.8 \%)$ \\
\hline \multicolumn{2}{|l|}{ CEUS indication $(n, \%)$} \\
\hline $\begin{array}{l}\text { Incidental finding in subjects without liver } \\
\text { pathology }\end{array}$ & $880(66.2 \%)$ \\
\hline $\begin{array}{l}\text { FLL in patients with chronic hepatopathies } \\
\text { (including liver cirrhosis) }\end{array}$ & $449(33.8 \%)$ \\
\hline \multicolumn{2}{|l|}{ Final diagnosis $(\mathrm{n}, \%)$} \\
\hline hepatocellular carcinoma (HCC) & $296(22.3 \%)$ \\
\hline metastasis & $346(26.1 \%)$ \\
\hline hemangiomas & $243(18.3 \%)$ \\
\hline focal fatty alterations & $175(13.2 \%)$ \\
\hline cholangiocarcinoma (CC) & $8(0.6 \%)$ \\
\hline focal nodular hyperplasia (FNH) & $55(4.1 \%)$ \\
\hline regenerating nodules & $82(6.2 \%)$ \\
\hline liver abscess & $29(2.2 \%)$ \\
\hline adenomas & $18(1.3 \%)$ \\
\hline complex cysts & $61(4.6 \%)$ \\
\hline hematomas & $5(0.3 \%)$ \\
\hline other benign lesions & $6(0.5 \%)$ \\
\hline other malignant lesions & $5(0.3 \%)$ \\
\hline \multicolumn{2}{|l|}{ The method used for the final diagnosis (n, \%) } \\
\hline CEUS & $1102(82.9 \%)$ \\
\hline CE-CT & $138(10.4 \%)$ \\
\hline CE-RMN & $82(6.2 \%)$ \\
\hline Biopsy & $7(0.5 \%)$ \\
\hline
\end{tabular}

The types of specific benign and malignant lesions diagnosed by CEUS are presented in Tables II and III.

CEUS performed significantly better for the diagnosis of hemangiomas (89.7\% cases diagnostic) and focal fatty alterations ( $89.7 \%$ cases diagnostic) than for the diagnosis of regenerative nodules ( $74.4 \%$ cases diagnostic) (Table IV), and also for the diagnosis of metastases ( $86.4 \%$ cases diagnostic) than of HCC (76.4\% cases diagnostic) and CC (25\% cases diagnostic), and for the diagnosis of HCC vs. CC, respectively (Table V).

Table II. Benign FLLs diagnosed by CEUS

\begin{tabular}{lccc}
\hline Benign FLLs & $\begin{array}{c}\text { Final diagnosis } \\
(\mathrm{n})\end{array}$ & $\begin{array}{c}\text { Diagnosed by } \\
\text { CEUS (n) }\end{array}$ & $\begin{array}{c}\text { Conclusive } \\
\text { at CEUS (\%) }\end{array}$ \\
\hline hemangioma & 243 & 218 & 89.7 \\
focal fatty alterations & 175 & 156 & 89.1 \\
$\begin{array}{l}\text { regenerative nodule } \\
\text { complex cyst }\end{array}$ & 82 & 61 & 74.4 \\
focal nodular & 61 & 53 & 86.9 \\
hyperplasia & 55 & 44 & 80 \\
abscess & 29 & 25 & 86.2 \\
hepatocellular & 18 & 14 & 77.8 \\
adenoma & & 3 & 60 \\
hematoma & 5 & & \\
\hline
\end{tabular}

Table III. Malignant FLLs diagnosed by CEUS

\begin{tabular}{lccc}
\hline Malignant FLLs & $\begin{array}{c}\text { Final diagnostic } \\
(\mathrm{n})\end{array}$ & $\begin{array}{c}\text { Diagnosed by } \\
\text { CEUS (n) }\end{array}$ & $\begin{array}{c}\text { Conclusive at } \\
\text { CEUS (\%) }\end{array}$ \\
\hline $\begin{array}{l}\text { metastasis } \\
\text { hepatocellular }\end{array}$ & 346 & 229 & 86.4 \\
$\begin{array}{l}\text { carcinoma } \\
\text { cholangio- } \\
\text { carcinoma }\end{array}$ & 296 & 226 & 76.4 \\
\hline
\end{tabular}

\section{DISCUSSION}

In the last 5-10 years, many papers have evaluated the accuracy of CEUS for FLL characterization. Why? Because CEUS is a rapid method of evaluation (it can be performed immediately after a FLL is found by US, so that the final diagnosis can be reached in approximately 5 minutes); it is quite inexpensive (2-5 times cheaper than CE-CT or CE-MRI) $[5,9-14]$; and, as opposed to CT examination, it is radiation free. On the other hand, the US contrast agent (SonoVue) is a safe drug, with very rare adverse events [15-19]. Since it is not excreted by the kidney (the micro-bubbles break and the gas is eliminated by the lung), it can be used in patients with kidney failure without causing kidney damage, as CE-CT contrast agents do.

Two pivotal multicentre studies that evaluated CEUS for the characterization of FLL, a German one (performed under the auspices of DEGUM - the German Society of Ultrasonography) [4] and a French one [5] showed the good value of this method for FLL characterization. The accuracy was slightly different in the German vs. the French study and also different when the accuracy in FLL on non-cirrhotic liver was compared to the one in lesions occurring in cirrhotic liver. On the other hand, 
Table IV. Comparison between the proportions of conclusive CEUS for different types of benign FLLs

\begin{tabular}{|c|c|c|c|}
\hline FLLs compared & $\mathrm{p}$ value & FLLs compared & p value \\
\hline hemangioma (89.7\%) vs. focal fatty alterations (89.1\%) & 0.97 & regenerative nodule $(74.4 \%)$ vs. focal nodular hyperplasia $(80 \%)$ & 0.57 \\
\hline hemangioma $(89.7 \%)$ vs. regenerative nodule $(74.4 \%)$ & 0.001 & regenerative nodule (74.4\%) vs. abscess $(86.2 \%)$ & 0.29 \\
\hline hemangioma ( $89.7 \%)$ vs. complex cyst $(86.9 \%)$ & 0.69 & regenerative nodule $(74.4 \%)$ vs. hepatocellular adenoma $(77.8 \%)$ & 0.99 \\
\hline hemangioma ( $89.7 \%)$ vs. focal nodular hyperplasia ( $80 \%)$ & 0.07 & regenerative nodule $(74.4 \%)$ vs. hematoma $(60 \%)$ & 0.85 \\
\hline hemangioma (89.7\%) vs. abscess (86.2\%) & 0.79 & complex cyst (86.9\%) vs. focal nodular hyperplasia ( $80 \%)$ & 0.45 \\
\hline hemangioma (89.7\%) vs. hepatocellular adenoma (77.8\%) & 0.27 & complex cyst $(86.9 \%)$ vs. abscess $(86.2 \%)$ & 0.8 \\
\hline hemangioma (89.7\%) vs. hematoma & 0.16 & complex cyst (86.9\%) vs. hepatocellular adenoma (77.8\%) & 0.56 \\
\hline focal fatty alterations $(89.1 \%)$ vs. regenerative nodule $(74.4 \%)$ & 0.004 & complex cyst $(86.9 \%)$ vs. hematoma $(60 \%)$ & 0.33 \\
\hline focal fatty alterations ( $89.1 \%)$ vs. complex cyst ( $86.9 \%)$ & 0.81 & focal nodular hyperplasia ( $80 \%$ ) vs. abscess $(86.2 \%)$ & 0.68 \\
\hline $\begin{array}{l}\text { focal fatty alterations }(89.1 \%) \text { vs. focal nodular hyperplasia } \\
(80 \%)\end{array}$ & 0.12 & $\begin{array}{l}\text { focal nodular hyperplasia }(80 \%) \text { vs. hepatocellular adenoma } \\
(77.8 \%)\end{array}$ & 0.89 \\
\hline focal fatty alterations ( $89.1 \%)$ vs. abscess $(86.2 \%)$ & 0.88 & focal nodular hyperplasia ( $80 \%$ ) vs. hematoma (60\%) & 0.63 \\
\hline $\begin{array}{l}\text { focal fatty alterations }(89.1 \%) \text { vs. hepatocellular adenoma } \\
(77.8 \%)\end{array}$ & 0.3 & abscess $(86.2 \%)$ vs. hepatocellular adenoma $(77.8 \%)$ & 0.72 \\
\hline focal fatty alterations $(89.1 \%)$ vs. hematoma $(60 \%)$ & 0.19 & abscess $(86.2 \%)$ vs. hematoma $(60 \%)$ & 0.43 \\
\hline regenerative nodule (74.4\%) vs. complex cyst (86.9\%) & 0.1 & hepatocellular adenoma $(77.8 \%)$ vs. hematoma $(60 \%)$ & 0.82 \\
\hline
\end{tabular}

Table V. Comparison between the proportions of conclusive CEUS for different types of malignant FLLs

\begin{tabular}{ll}
\hline FLLs compared & $\mathrm{p}$ value \\
\hline metastasis $(86.4 \%)$ vs. hepatocellular carcinoma (76.4\%) & 0.001 \\
metastasis (86.4\%) vs. cholangiocarcinoma (25\%) & $<0.0001$ \\
hepatocellular carcinoma (76.4\%) vs. cholangiocarcinoma & 0.003 \\
$(25 \%)$ & \\
\hline
\end{tabular}

the accuracy of FLL characterization differs for various types of liver lesions, being higher for hemangiomas or metastasis and lower for hepatocellular carcinomas or adenomas.

The DEGUM study [4] was performed in Germany over 20 months and included 1349 patients with undiagnosed FLL on US. A number of 1328 FLLs were assessed, 755 of them malignant and 573 benign. In $75 \%$ of the cases the final diagnosis was established by liver biopsy, while in other cases the "gold-standard" method was CE-CT or CE-MRI.

The French study (STIC) [5] assessed the clinical value of CEUS using SonoVue ${ }^{\circ}$ for the characterization of FLLs discovered in patients with a cancer history, or in those with chronic liver disease. The study included 874 patients in whom 1,034 FLLs, undiagnosed based only on US, were analyzed. The "gold-standard" methods to which CEUS was compared were either CE-CT, CE-MRI, or liver biopsy. In this study, CEUS had good sensitivity $(79.4 \%)$ and specificity (88.1\%) to differentiate benign vs. malignant FLLs.

In our study, we intended to evaluate the results of CEUS for the characterization of FLLs in a large monocentric experience (the previously mentioned studies were multicenter ones), in order to decide if CEUS is suitable to be used as a first line imaging diagnostic procedure. The first information that CEUS should give is if the lesion assessed is benign or malignant (based on washout occurrence in the portal and late phases) and secondly, to establish the exact type of FLL based on the enhancement pattern following contrast. Since our purpose was not to demonstrate the accuracy of CEUS (already demonstrated in numerous studies) we performed additional studies (CE-CT, CE-MRI, liver biopsy) only when CEUS was not conclusive, thus explaining the small number of liver biopsies (only in $0.5 \%$ of cases): these were performed only in cases that could not be diagnosed by non-invasive imaging methods.

In the last 10 years, numerous papers have been published (both monocentric and multicenter trials) that showed the value of CEUS for FLLs characterization, all of them with good results, confirming the usefulness of CEUS as a first line diagnostic procedure when a lesion is discovered in US. This strategy saves time and stress for the patients, it saves money and it is a safe procedure. Also several papers were published comparing CEUS with CE-CT or CE-MRI $[5,7,20]$ for the diagnosis of FLLs (such as metastasis, FNH or hemangiomas), showing similar results. A recent meta-analysis performed by Friedrich Rust et al [8] demonstrated that CEUS offers a good performance to determine the benign and malignant nature of a FLL.

In our study we learned that CEUS is able to differentiate benign vs. malignant lesions in $90 \%$ of cases, our results being similar to other already published studies [7, 8, 21-24]. Based on the results of many studies $[4,5,7,8,25-30]$, CEUS seems to be a reliable method for a good characterization of FLL, this being observed also by us. But we must underline that we found significant statistical differences between the proportions of conclusive CEUS comparing the cohorts of patients with or without chronic hepatopathies. What is the explanation? In patients with liver cirrhosis, in many cases, the liver structure is heterogeneous. The homogeneous US aspect of the healthy liver facilitates the use of CEUS for FLL characterization. Sometimes, CEUS is difficult to perform, or even impossible, in deeply situated liver lesions and thus, for FLLs located at 7 $\mathrm{cm}$ or deeper under the liver surface, CEUS should be avoided [2]. Also, this technique must be performed by an experienced examiner using a performant system. Therefore, this method is not available to everyone, this being one of its limits. 
Considering the FLL size, we found that the percentage of cases with a conclusive diagnosis was not significantly different in lesions smaller than $2 \mathrm{~cm}$ or larger, such as in the DEGUM study [4]. One explanation can be that even if in small HCCs the CEUS diagnosis is more difficult, in other lesions (such as liver metastases, FNH or hemangiomas) CEUS is able to establish a positive diagnosis despite the lesion size.

So what is the main purpose of a physician performing CEUS? Firstly to establish the benign or malignant nature of the lesion and secondly to decide if a final diagnosis regarding the type of lesion can be established based on the enhancement pattern. For these two purposes, CEUS seems to be a good method in a center with a large experience. If these two questions are not answered, the physician must recommend the patient to undergo another diagnostic method. Usually a discussion with the radiologist will decide, in the clinical context, which is the next imaging method that should be used. Sometimes, even CE-CT and CE-MRI are not able to establish a final diagnosis and in this case a US-guided biopsy must be performed. This strategy seems to be the most cost effective [31-34].

The weakness of this study is that we did not use a "gold standard" method to evaluate our results. However, it was not our intention to do that since the value of CEUS for the diagnosis of FLLs has been proven in large multicenter studies and meta-analyses, and also because three EFSUMB Guidelines (one in cooperation with WFUMB) have been published. It is thus the moment to use this method similarly to CE-CT or CE-MRI, and when the lesion has a typical enhancement pattern, to establish the final diagnosis. For an expert team, it is easy to decide if the aspect of the lesion is conclusive or not and, when required, to ask for another imaging method.

\section{CONCLUSION}

The CEUS patterns of enhancement fell into clear cut specific diagnostic patterns in $83 \%$ of the FLLs discovered by US, and into clear cut benign versus malignant patterns in $90 \%$ of the cases. For this reason, we strongly recommend CEUS as a first line imaging method to characterize FLLs found at US, at least in centers with a good experience in CEUS.

Conflicts of interest. No conflict to declare.

\section{REFERENCES}

1. Albrecht T, Blomley M, Bolondi L, et al; EFSUMB Study Group. Guidelines for the use of contrast agents in ultrasound. January 2004. Ultraschall Med 2004;25:249-256.

2. Claudon M, Cosgrove D, Albrecht T, et al. Guidelines and good clinical practice recommendations for contrast enhanced ultrasound (CEUS) - update 2008. Ultraschall Med 2008;29:28-44.

3. Claudon M, Dietrich CF, Choi BI, et al. Guidelines and good clinical practice recommendations for Contrast Enhanced Ultrasound (CEUS) in the liver - update 2012: A WFUMB-EFSUMB initiative in cooperation with representatives of AFSUMB, AIUM, ASUM, FLAUS and ICUS. Ultrasound Med Biol 2013;39 187-210.

4. Strobel D, Seitz K, Blank W, et al. Contrast-enhanced ultrasound for the characterization of focal liver lesions-diagnostic accuracy in clinical practice (DEGUM multicenter trial). Ultraschall Med 2008;29:499505.

5. Tranquart F, Le Gouge A, Correas JM, et al. Role of contrast-enhanced ultrasound in the blinded assessment of focal lesions in comparison with MDCT and CEMRI: Results from a multicentre clinical trial. EJC Supplements 2008;6:9-15.

6. Sporea I, Badea R, Martie A, et al. Contrast Enhanced Ultrasound for the evaluation of focal liver lesions in daily practice. A multicentre study. Med Ultrason 2012;14:95-100.

7. Xie L, Guang Y, Ding H, Cai A, Huang Y. Diagnostic value of contrastenhanced ultrasound, computed tomography and magnetic resonance imaging for focal liver lesions: a meta-analysis. Ultrasound Med Biol 2011;37:854-861.

8. Friedrich-Rust M, Klopffleisch T, Nierhoff J, et al. Contrast Enhanced Ultrasound for the differentiation of benign and malignant focal liver lesions: a meta-analysis. Liver Int 2013;33:739-755.

9. Giesel FL, Delorme S, Sibbel R, Kauczor HU, Krix M. Contrast-enhanced ultrasound for the characterization of incidental liver lesions- an economical evaluation in comparison with multi-phase computed tomography. Ultraschall Med 2009;30:259-268.

10. Piscaglia F, Leoni S, Cabibbo G, et al. Cost analysis of recall strategies for non-invasive diagnosis of small hepatocellular carcinoma. Dig Liver Dis 2010;42:729-734.

11. Faccioli N, D’Onofrio M, Comai A, Cugini C. Contrast-enhanced ultrasonography in the characterization of benign focal liver lesions: activity-based cost analysis. Radiol Med 2007;112:810-820.

12. Romanini L, Passamonti M, Aiani L, et al. Economic assessment of contrast-enhanced ultrasonography for evaluation of focal liver lesions: a multicentre Italian experience. Eur Radiol 2007;17 Suppl 6:F99-F106.

13. Şirli R, Sporea I, Martie A, Popescu A, Dănilă M. Contrast enhanced ultrasound in focal liver lesions -a cost efficiency study. Med Ultrason 2010;12:280-285.

14. NHS National Institute for Health and Clinical Excellence. SonoVue (sulphur hexafluoride microbubbles) - contrast agent for contrastenhanced ultrasound imaging of the liver. NICE diagnostics guidance 5. NICE 2012. www.nice.org.uk/dg5.

15. Piscaglia F, Bolondi L; Italian society for ultrasound in medicine and biology (SIUMB) study group on Ultrasound Contrast Agents. The safety of Sonovue in abdominal applications: retrospective analysis of 23188 investigations. Ultrasound Med Biol 2006;32:1369-1375.

16. Gaibazzi N, Squeri A, Ardissino D, Reverberi C. Safety of contrast flash-replenishment stress echocardiography in 500 patients with a chest pain episode of undetermined origin within the last 5 days. Eur J Echocardiogr 2009;10:726-732.

17. Bokor D, Chambers J B, Rees P J, Mant TG, Luzzani F, Spinazzi A. Clinical safety of SonoVue, a new contrast agent for ultrasound imaging, in healthy volunteers and in patients with chronic obstructive pulmonary disease. Invest Radiol 2001;36:104-109.

18. Beaton C, Cochlin D, Kumar N. Contrast enhanced ultrasound should be the initial radiological investigation to characterise focal liver lesions. Eur J Surg Oncol 2010;36:43-46.

19. Jakobsen JA, Oyen R, Thomsen HS, et al. Safety of ultrasound contrast agents. Eur Radiol 2005;15:941-945.

20. Westwood M, Joore M, Grutters J, et al. Contrast-enhanced ultrasound using SonoVue ${ }^{\circ}$ (sulphur hexafluoride microbubbles) compared with contrast-enhanced computed tomography and contrast-enhanced magnetic resonance imaging for the characterisation of focal liver lesions and detection of liver metastases: a systematic review and costeffectiveness analysis. Health Technol Assess 2013;17:1-243. 
21. von Herbay A, Westendorff J, Gregor M. Contrast-enhanced ultrasound with SonoVue: differentiation between benign and malignant focal liver lesions in 317 patients. J Clin Ultrasound 2010;38:1-9.

22. Ooi CC, Low SC, Schneider-Kolsky M, et al. Diagnostic accuracy of contrast-enhanced ultrasound in differentiating benign and malignant focal liver lesions: a retrospective study. J Med Imaging Radiat Oncol 2010;54:421-430.

23. Quaia E, Alaimo V, Baratella E, et al. Effect of observer experience in the differentiation between benign and malignant liver tumors after ultrasound contrast agent injection. J Ultrasound Med 2010;29:2536.

24. Celli N, Gaiani S, Piscaglia F, et al. Characterization of liver lesions by real-time contrast-enhanced ultrasonography. Eur J Gastroenterol Hepatol 2007;19:3-14.

25. Dai Y, Chen MH, Fan ZH, Yan K, Yin SS, Zhang XP. Diagnosis of small hepatic nodules detected by surveillance ultrasound in patients with cirrhosis: Comparison between contrast-enhanced ultrasound and contrast-enhanced helical computed tomography. Hepatol Res 2008;38:281-290.

26. Liu LP, Dong BW, Yu XL, Zhang DK, Kang CS, Zhao XH. Evaluation of focal fatty infiltration of the liver using color Doppler and contrastenhanced sonography. J Clin Ultrasound 2008;36:560-566.
27. Piscaglia F, Venturi A, Mancini M, et al. Diagnostic features of real-time contrast-enhanced ultrasound in focal nodular hyperplasia of the liver. Ultraschall Med 2010;31:276-282.

28. Sirli R, Sporea I, Popescu A, et al. Contrast enhanced ultrasound for the diagnosis of liver hemangiomas in clinical practice. Med Ultrason 2011;13:95-101.

29. Cabassa P, Bipat S, Longaretti L, Morone M, Maroldi R. Liver metastases: Sulphur hexafluoride-enhanced ultrasonography for lesion detection a systematic review. Ultrasound Med Biol 2010;36:1561-1567.

30. Larsen LP. Role of contrast enhanced ultrasonography in the assessment of hepatic metastases: A review. World J Hepatol 2010;2:8-15.

31. Pasha T, Gabriel S, Therneau T, Dickson ER, Lindor KD. Costeffectiveness of ultrasound-guided liver biopsy. Hepatology 1998;27:1220-1226.

32. Shah S, Mayberry J, Wicks A, Rees Y, Playford RJ. Liver biopsy under ultrasound control: implications for training in the Calman era. Gut 1999;45:628-629.

33. Arguedas MR, Chen VK, Eloubeidi MA, Fallon MB. Screening for hepatocellular carcinoma in patients with hepatitis $\mathrm{C}$ cirrhosis: a costutility analysis. Am J Gastroenterol 2003;98:679-690.

34. Iavarone M, Manini MA, Sangiovanni A, et al. Contrast-enhanced computed tomography and ultrasound-guided liver biopsy to diagnose dysplastic liver nodules in cirrhosis. Dig Liver Dis 2013;45:43-49. 